\title{
7 Induced Mutagenesis and Quantitative Traits
}

For the last four decades the practical value of induced mutagenesis, in creating successful genetic variability for several desired traits in plant improvement programmes, has been well established and has been demonstrated by many workers in different crop categories viz. pulses like pigeonpea (Rao, 1984; Srivastava and Singh, 1993), cowpea (Murugan and Subramanian, 1993; Gunasekaran et al., 1998; Pandey, 2002), urdbean (Singh and Singh, 2001; Selvam et al., 2010), lentil (Kumar and Lal, 2001; Solanki and Sharma, 2001, 2002; Khan et al., 2006b), faba bean (Filippetti and De Pace, 1986; Verma and Rao, 1994; Joshi and Verma, 2004), mungbean (Khan, 1984; Singh and Yadav, 1991; Mathew et al., 2005; Khan and Goyal, 2009), chickpea (Harer et al., 1999; Kharkwal, 2001; Khan and Wani, 2005b, Kozgar and Khan, 2009), cereals like wheat (Scossiroli, 1964; Konzak, 1973; Siddiqui, 1983; Boreiko et al., 1986; Khan, 1988; Nalini et al., 1993; Kalia et al., 2000; Jamil and Khan, 2002; Sakin and Yildirim, 2004), rice (Swaminathan et al., 1969; Misra et al. 1973; Awan et al., 1980; Shanthi and Singh, 2001; Ishiy et al., 2006), barley (Gustafsson, 1963; Gaul, 1964; Bhargava and Khalatkar, 1986; Nalini et al., 1993), triticale (Reddy, 1988, 1989; Viswanathan et al., 1994), other ornamental and medicinal plants (Amarnath and Prasad, 2000; Cagirgan, 2006; Datta and Laxmi, 1992; Datta and Sengupta, 2002; Kumar et al., 1996; Marry and Jayabalan, 1995). In all the cases, most of the plant attributes of interest to plant breeders were quantitative traits which are governed by the principles of quantitative genetics.

A common practice in mutation breeding programme is to advance only normal looking $M_{2}$ plants to $M_{3}$ generation, and apply the first dose of selection in $M_{2}$ generation (Brock, 1965; 1967). This methodology has been advocated by Gupta and Swaminathan (1967), Tickoo and Jain (1979), Sharma (1986) and Wani and Khan (2006), however, Jana and Roy (1973) selected $\mathrm{M}_{2}$ families on the basis of significantly changed mean only. Since most of the desired combinations of favourable alleles are likely to be lost in advanced generations due to intensive or even no selection for other traits thus the selection for quantitative traits, such as yield and yield attributing characters, should preferably be carried out in early generation (Sneep, 1977; Saini and Gautam, 1990 and Sharma, 1997). The efficiency of early generation $\left(M_{2}\right)$ selection in mutation breeding experiments has been reported in the crops like lentil (Solanki and Sharma, 2002), pea (Singh, 1988), mungbean (Tickoo and Chandra, 1999), sesame (Sheeba et al., 2003), soybean (Pavadai et al., 2010; Nakagawa, 2009 and Nakagawa et al., 2011). Since the quantitative traits selected in early generations indicate the degree of stability to the environmental fluctuations, henceforth the potential transmissibility of these traits from parent to offsprings, and from generation to generation, are to be evaluated. For this, the estimates of heritability of various quantitative traits are essential (Mather and Jinks, 1971; Kaul and Garg, 1979, Scossiroli et al., 1966; Ignacimuthu and Babu, 1993; Brunner, 1995; Mohanty, 2001; Chaudhary et al., 2004; Khan et al., 2006; Arulbalachandran et al., 2010). Trivedi et al. (2006) clearly brought 
out that, in the treated population, the estimates of heritability were larger and varied from trait to trait. Johnson et al. (1955) suggested that heritability in combination from genetic advance was more helpful in predicting the effect of selection. This has been advocated by many workers like Kaul and Garg (1979), Sakin, (2002) and Khan and Goyal (2009). Higher values of heritability and genetic advance over the control population for certain quantitative traits were reported by Kaul and Kumar (1983) and Rather et al. (1998) in rice, Sharma and Sharma (1984) in lentil, Mehetre et al. (1998) in soybean, Sakin and Yildirim (2004) in wheat, Khan and Wani (2006) in green gram and Mohammadi and Pourdad (2009) in safflower.

Both physical and chemical mutagens, alone or in combinations, were used in pulses for generating variability in quantitative traits like plant height, pods per plant, pod length, flowering and maturity period, seed weight, biological yield, number of fertile branches, number of seeds per pod, yield and harvest index (Kundu and Singh, 1981, Kundu and Singh, 1982; Reddy et al., 1992; Srivastava and Singh, 1993; Kumar et al., 1995; Waghmare and Mehra, 2000; Rehman et al., 2001; Yaqoob and Rashid, 2001, Wani and Khan 2004, Wani et al., 2011c). The important primary yield components in pulses are considered pods bearing branches, pods per plant, seeds per pod and seed weight and several studies (Bahl, 1988; Kumar and Arora, 1991; Rao, 1996; Khan and Siddiqui, 1997; Guler et al., 2001; Kharkwal, 2003; Singh and Singh, 2003; Raut et al., 2004; Khan and Wani, 2005b; Mubeen et al., 2007; Makeen et al., 2009; Giri et al., 2010) have shown a close association between these components to the total plant yield in induced mutant lines. In chickpea, different workers have reported increased variability for various agronomic characters in mutagen treated population as observed by significant changes in the mean values and coefficient of variability as compared to control (Mandal, 1974; Nerker and Mote, 1978; Haq and Shakoor, 1980; Kharkwal, 1981; Kumar et al., 1981; Kozgar and Khan, 2009; Barshille et al., 2009; Kozgar et al. 2012).

\subsection{Case Study 5}

In $M_{2}$ generation, the observations were made on 25-30 normal looking plants of each progeny, for each treatment along with the control (see Appendix materials and methods). The progenies segregating for macromutations were not used for such analysis. The following eight quantitative traits were thoroughly studied in different generations:

(i) Days to flowering; (ii) Plant height; (iii) Days to maturity; (iv) Number of pods bearing branches; (v) Number of pods; (vi) Seeds per pod; (vii) 100-seed weight; (viii) Total Seed Plant Seed Yield.

For raising $\mathrm{M}_{3}$ generation, two treatments of gamma rays and EMS alone and their combinations for each variety were used which gave the maximum total plant yield in $\mathrm{M}_{2}$ generation. 


\subsubsection{Results}

The availability of ample genetic variability particularly for quantitatively inherited traits is a pre-requisite for attempting selection in plant breeding to develop ideotypes in self pollinating crops like chickpea.

The genetic variability induced for eight quantitative traits by gamma rays, EMS and their combination treatments was studied in the two varieties of chickpea viz. Pusa-256 and BG-1053 in $M_{2}$ and $M_{3}$ generations. Since, the genetic parameters such as genotypic coefficient of variation (GCV), heritability in broad sense $\left(\mathrm{h}^{2}\right)$ and genetic advance as percentage of mean (GA) provide guideline for the improvement of quantitative traits, their estimates were determined for each trait in the mutagenized population. Results obtained for each trait are elaborated below and examplenary alterations in genetic advances are given in Table 7.1 with respect to mutagen type:

\section{(i) Days to flowering}

The procured results on mean values, shift in mean values, genotypic coefficient of variation, heritability and genetic advance for days to flowering in $M_{2}$ generation showed that the mean values shifted negatively in the mutagenized population. The mean days to flowering decreased significantly by three days with the treatments of $0.4 \%$ EMS in the Pusa-256. Though the doses were increasing in a linear order, yet the induced genotypic coefficient of variation did not show a consistent increase. The highest genotypic variability was recorded with 0.4\% EMS treatments in the variety Pusa-256 while the highest genotypic variability was recorded with $100 \mathrm{~Gy}$ of gamma rays and 200 Gy gamma rays + 0.1\% EMS combination treatments in the variety BG-1053 (unpublished data).

Heritability and genetic advance showed a considerable increase over the control in both varieties. In most of the mutagenic treatments, higher estimates of heritability are associated with maximum genetic advance. The highest heritability estimates were recorded in the variety Pusa-256 with 0.3\% EMS, whereas in the variety BG-1053, it was the highest in combination treatment of 200 Gy gamma rays $+0.1 \%$ EMS.

The maximum genetic advance in the treated population of the variety Pusa-256 was with $0.4 \%$ EMS, while it was recorded to be the highest (12.34\%) $200 \mathrm{~Gy}$ gamma rays $+0.1 \%$ EMS combination treatment in the variety BG-1053.

In $\mathrm{M}_{3}$ generation the mean flowering time was reduced for all the treatments in both varieties. The mean values of the treated population differed significantly from the controls. However, $200 \mathrm{~Gy}$ gamma rays, $100 \mathrm{~Gy}$ gamma rays + 0.1\% EMS and EMS treatments had no significant effect on the mean flowering time in the variety BG-1053.

The estimated values of genotypic coefficients of variation, heritability and genetic advance were slightly decreased as compared to $M_{2}$ generation but were still higher than the controls of both varieties. 
Table 7.1: Tabulated format of Genetic Advance (as \% of $\bar{X}$ ) alterations due to mutagen induced mutagenesis in two varieties of case studied chickpea in $M_{3}$ generation.

\begin{tabular}{lllllll}
\hline Feature & \multicolumn{3}{c}{ Pusa-256 } & \multicolumn{3}{c}{ BG-1053 } \\
\cline { 2 - 6 } & gamma ray & EMS & $\begin{array}{l}\text { gamma ray } \\
\text { + EMS }\end{array}$ & gamma ray EMS & $\begin{array}{l}\text { gamma ray } \\
\text { + EMS }\end{array}$ \\
\hline Days to flowering & $\uparrow(5.5 \%)$ & $\uparrow(7.0 \%)$ & $\uparrow(7.5 \%)$ & $\uparrow(4.0 \%)$ & $\uparrow(7.0 \%)$ & $\uparrow(5.0 \%)$ \\
Plant height & $\uparrow(8.0 \%)$ & $\uparrow(6.0 \%)$ & $\uparrow(5.5 \%)$ & $\uparrow(15.0 \%)$ & $\uparrow(6.5 .0 \%)$ & $\uparrow(6.0 \%)$ \\
Days to maturity & $\uparrow(3.0 \%)$ & $\uparrow(4.0 \%)$ & $\uparrow(4.0 \%)$ & $\uparrow(4.5 \%)$ & $\uparrow(5.5 \%)$ & $\uparrow(6.5 \%)$ \\
Number of Pods & $\uparrow(45.0 \%)$ & $\uparrow(45.0 \%)$ & $\uparrow(47.5 \%)$ & $\uparrow(44.0 \%)$ & $\uparrow(55.0 \%)$ & $\uparrow(44.5 \%)$ \\
Bearing Branches & & & & & & \\
Number of pods per plant & $\uparrow(41.0 \%)$ & $\uparrow(50.0 \%)$ & $\uparrow(40.5 \%)$ & $\uparrow(39.5 \%)$ & $\uparrow(41.0 \%)$ & $\uparrow(40.5 \%)$ \\
Number of Seeds per Pod & $\uparrow(23.0 \%)$ & $\uparrow(28.0 \%)$ & $\uparrow(33.0 \%)$ & $\uparrow(23.0 \%)$ & $\uparrow(28.0 \%)$ & $\uparrow(24.5 \%)$ \\
100 Seed Weight & $\uparrow(38.0 \%)$ & $\uparrow(39.0 \%)$ & $\uparrow(34.5 \%)$ & $\uparrow(41.0 \%)$ & $\uparrow(28.0 \%)$ & $\uparrow(20.5 \%)$ \\
Total plant seed yield & $\uparrow(29.0 \%)$ & $\uparrow(27.0 \%)$ & $\uparrow(36.0 \%)$ & $\uparrow(28.0 \%)$ & $\uparrow(35.5 .0 \%) \uparrow(33.0 \%)$ \\
\hline
\end{tabular}

\section{(ii) Plant height (cm)}

Results recorded on plant height indicate that the mean values shifted significantly in the negative direction in most of the treatments with gamma rays and EMS alone or in combination in $\mathrm{M}_{2}$ generation. Plant height showed a greater reduction in the combined treatments compared to individual mutagenic treatments of gamma rays and EMS. The genotypic coefficient of variation was higher in all the treatments. The highest genotypic coefficient of variation was at $400 \mathrm{~Gy}$ of gamma rays and at 0.3\% EMS as observed in the varieties Pusa-256 and BG-1053, respectively.

Heritability and genetic advance increased in both single and combined treatments in both varieties. The highest heritability for both varieties was recorded with the treatment of $400 \mathrm{~Gy}$ gamma rays. The highest values of genetic advance were recorded with $400 \mathrm{~Gy}$ gamma rays in the variety Pusa-256 and with the treatment of $0.2 \%$ EMS in the variety BG-1053.

The mean values for plant height in $M_{3}$ were significantly reduced in all the treatments of gamma rays and EMS employed alone or in combination in both varieties. The reduction in mean plant height was the highest in combination treatments of gamma rays + EMS.

The genotypic coefficient of variation, heritability and genetic advance increased in almost all treatments. The highest genotypic coefficient of variation was recorded in the gamma rays treated population. The highest heritability estimate was obtained with 300 Gy of gamma rays treatment in the variety BG-1053. The genetic advance was similarly affected. Genetic parameters were recorded lower in $M_{3}$ than in $M_{2}$ generation. 


\section{(iii) Days to maturity}

The results obtained on the effect of single and combination treatments of gamma rays and EMS on days to maturity in $\mathrm{M}_{2}$ generations revealed that the mean for days to maturity was shifted towards the negative side in all the mutagen treatments in both the varieties. The highest reduction in mean was observed in the variety Pusa256 with 200 Gy gamma rays + 0.1\% EMS treatment followed by 200 Gy gamma rays $+0.2 \%$ EMS treatment in the variety BG-1053. Reduction in the mean was higher in combination treatments as compared to individual mutagenic treatments of gamma rays and EMS.

The genotypic coefficient of variation increased considerably in most of the treatments. The estimated GCV was the highest with $200 \mathrm{~Gy}$ gamma rays + 0.1\% EMS in the variety Pusa-256 whereas, in the variety BG-1053, the highest GCV was obtained with $0.2 \%$ EMS treatment.

The estimated heritability shows considerable variation for days to maturity and was the highest in under study varieties of chickpea at $200 \mathrm{~Gy}$ gamma rays $+0.1 \%$ EMS combined treatment. The highest genetic advance was recorded with $200 \mathrm{~Gy}$ gamma rays + 0.1\% EMS treatment in the variety Pusa-256 and with 0.2\% EMS in the variety BG-1053.

The days to maturity in $M_{3}$ generation in most of the single and combination treatments of gamma rays and EMS was significantly reduced. Early maturity by 4 days was recorded with $0.3 \%$ EMS and 100 Gy gamma rays + $0.2 \%$ EMS treatments in the variety Pusa-256.

The genotypic coefficient of variation, heritability and genetic advance were recorded higher in the treated population. The genetic parameters did not show much variation in any of the treatments used when compared to control. The values of genetic parameters were lower in $\mathrm{M}_{3}$ than $\mathrm{M}_{2}$ generation.

\section{(iv) Number of pods bearing branches}

The results recorded in $\mathrm{M}_{2}$ generation on number of pods bearing branches per plant showed that the mean shifts in the positive direction in all mutagen treatments, except for the doses of gamma rays, EMS and their combination, in the variety BG-1053. In general, there was an increasing trend of mean values of number of pods bearing branches in almost all mutagens in both varieties.

The genotypic coefficient of variation increased with all the treatments in both varieties. The maximum GCV in both varieties of chickpea under consideration was recorded with $0.2 \%$ of EMS treatment.

High heritability coupled with high genetic advance was observed for number of pods bearing branches in both varieties. The heritability and genetic advance were maximum with 300 Gy gamma rays treatment in the variety Pusa-256. On the other hand, the $0.2 \%$ of EMS treatment gave the maximum heritability and genetic advance in the variety BG-1053. 
The data on the number of the pods bearing branches per plant in $M_{3}$ generation showed significant increase in mean values of the pods bearing branches in all the mutagen treatments in both varieties.

The genotypic coefficient of variation increased in the mutagenized population. The most effective treatment with regard to heritability and genetic advance was $300 \mathrm{~Gy}$ gamma rays in the variety Pusa-256. As to the variety BG-1053 the most effective treatment was $0.2 \%$ EMS, which gave the maximum heritability and genetic advance.

\section{(v) Number of pods per plant}

The data recorded on pods per plant in $\mathrm{M}_{2}$ generation revealed the mean value shifted in both positive as well as in negative direction. Decrease in mean values, compared to the control, was mostly found at the higher doses of the mutagens used alone or in combination. The lower and moderate doses of mutagens significantly increased the mean number of pods per plant. The mean values increased more in the variety Pusa256 than in the variety BG-1053 (unpublished data).

The genotypic coefficient of variation was recorded to be higher with all the treatments of mutagens. The highest GCV was recorded with $100 \mathrm{~Gy}$ gamma rays + 0.1\% EMS for variety Pusa-256 and 100 Gy gamma rays treatments for variety BG-1053.

The heritability and genetic advance showed considerable variation for the number of pods per plant in both varieties. The estimated heritability and genetic advance were the highest at 100 Gy gamma rays + 0.1\% EMS in the variety Pusa-256. The variety BG-1053 showed the maximum heritability and genetic advance with $0.3 \%$ EMS treatment.

The mean values for the number of pods per plant increased over the controls in both varieties for all mutagenic treatments selected in $M_{3}$ generation. The mean values for the treated population differed significantly from the control mean.

The genetic parameters were elevated in all the treatments of gamma rays and EMS employed alone or in combination. The highest genotypic coefficient of variation, heritability and genetic advance was observed in the combination treatment of $100 \mathrm{~Gy}$ gamma rays $+0.1 \%$ EMS in the variety Pusa-256. The values of such genetic parameters were the highest with $0.2 \%$ EMS in the variety BG-1053. High heritability coupled with high genetic advance was recorded in most of the single and combination treatments of gamma rays and EMS.

\section{(vi) Number of seeds per pod}

The seeds per pod in the two varieties showed that most of the single and combination treatments of gamma rays and EMS were not capable of inducing significant differences in the mean number of seeds per pod. The mean number of seeds per pod for the varieties Pusa-256 and BG-1053 were the highest with 200 Gy gamma rays treatment.

The estimated genotypic coefficient of variation for the variety Pusa-256 was the highest in combination treatment of $100 \mathrm{~Gy}$ gamma rays + 0.1\% EMS. GCV for the variety BG-1053 was the highest at $200 \mathrm{~Gy}$ gamma rays. It is interesting to note that in most of the 
treatments the heritability percentage was lower than in the control in both varieties. However, the genetic advance increased for most of the treatments of gamma rays and EMS alone or in combination. The genetic advance estimates in the variety Pusa-256 were the highest with $100 \mathrm{~Gy}$ gamma rays + $0.1 \%$ EMS treatment whereas in the variety B-1053, the highest value was found in $200 \mathrm{~Gy}$ gamma rays treatments.

The mean values of the treated population did not differ significantly from the control in most of the mutagenic treatments of the $\mathrm{M}_{3}$ generation raised plants.

As regards the genetic parameters, the variability was higher in the combined treatments of gamma rays + EMS followed by EMS and gamma rays treatments. However, estimated heritability was lower in comparison to control in number of treatments. The genetic advances increased in both single and combined treatments except 300 Gy gamma rays in the variety Pusa-256.

\section{(vi) 100 seed weight (g)}

The data recorded for 100 seed weight $(\mathrm{g})$ after various mutagenic treatments depicts that the shift in mean values goes bi-directional, being more in the positive direction. Mean values for 100 seed weight increased significantly at almost all the lower doses of mutagens. However, the most effective treatments for increase in mean values of 100 seed weight were recorded for $200 \mathrm{~Gy}$ and $300 \mathrm{~Gy}$ gamma rays, $0.1 \%$ and $0.2 \%$ EMS and the combined treatments of 100 Gy gamma rays + 0.1\% EMS and $100 \mathrm{~Gy}$ gamma rays $+0.2 \%$ EMS. There was a significant reduction in 100 seed weight at the higher doses of gamma rays and EMS employed alone, or in combination, with a few exceptions.

The genotypic coefficients of variation were higher in the treated population as compared to controls. The highest GCV was recorded in combination treatment of 100 Gy gamma rays + 0.2\% EMS in the variety Pusa-256 while the highest estimated GCV was obtained at $0.3 \%$ EMS in the variety BG-1053.

The estimated heritability and genetic advance were the highest in combination treatments of 100 Gy gamma rays + 0.2\% EMS in the variety Pusa-256 while the variety BG-1053 gave the highest estimated heritability and genetic advances with $0.3 \%$ EMS.

Results recorded for 100 seed weight showed that the mean values increased significantly with each treatment, except in the combined treatments of gamma rays + EMS in the variety Pusa-256, of both varieties in $\mathrm{M}_{3}$ generations.

The genetic parameters increased in the treated population as compared to the controls. The values of genotypic coefficient of variation, heritability and the genetic advance were the highest at $0.2 \%$ EMS treatment in the variety Pusa-256, whereas in the variety BG-1053, the highest values of genotypic coefficient of variation, heritability and the genetic advance were recorded with $200 \mathrm{~Gy}$ gamma rays treatment.

\section{(viii) Total plant seed yield (g)}

The results recorded on seed yield per plant presented here revealed that the mean value shifted in both positive as well as in negative directions in the treated population. 
In general, the lower and moderate doses of gamma rays and EMS and the lower doses of gamma rays + EMS combined treatments showed a significant increase in mean yield per plant over the controls in both varieties. The mean values increased more in the variety Pusa-256 than in the variety BG-1053.

The genotypic coefficient of variation, heritability and genetic advance increased over the controls with all the treatments in both varieties. In the variety Pusa-256, the highest GCV, heritability and genetic advance were recorded at $0.3 \% \mathrm{EMS}$, whereas GCV, heritability and genetic advance were the highest in combination treatments of 100 Gy gamma rays + 0.1\% EMS in the variety BG-1053.

The high heritability coupled with high genetic advance was recorded for number of the treatments with gamma rays, and EMS alone, or in combination, indicating that significant gains could be expected from selection.

Mutagen treatments, selected for $M_{3}$ generation, exhibited an increase in the mean values for total plant seed yield in both selected varieties of chickpea in $M_{3}$ generations. The mean values in the treated population significantly differed from these in the control.

The genetic parameters for total plant yield increased in the treated population as compared to control. The highest genotypic coefficient of variation and genetic advance were observed with 300 Gy gamma rays treatment in the variety Pusa-256, whereas in the variety BG-1053, 100 Gy gamma rays + 0.1\% EMS treatment gave the highest values of genetic parameters.

\subsubsection{Discussion}

It is now the known fact that the availability of the large genetic variability within the species is prerequisite for the improvement of the cultivated plants and the mutagenesis has proved to be a handy tool to enhance the mutation rate and thereby enlarging the genetic variability and increasing the scope for obtaining the desired selections. Particularly, induction of micromutations in the polygenic system, controlling the quantitative traits is important for crop improvement. From the work already reported by several authors in various crops (Gregory, 1965; Swaminathan, 1969; Borojevic and Borojevic, 1972; Ignacimuthu and Babu, 1993; Khan et al., 1998; Joshi and Verma, 2004; Shin et al., 2011) it is now quite clear that micromutations result in the release of considerable genetic variability in the mutagen treated population. In some studies on the use of mutations for quantitative traits improvement, it was found that different traits differ in their response to the mutagenic treatments. Variance level may be less responsive in one trait and highly responsive in other (Kaul and Kumar, 1983; Sharma, 1995; Khan and Wani, 2006a). Moreover, the direction of polygenic mutations depends on the genotypic background of the material under study (Loesch, 1964). Thus, the genetic improvement of such traits in turn depends upon the magnitude of genetic parameters and the breeding methodology adopted. 
Estimates of genetic parameters like genotypic coefficient of variation, heritability and genetic advance are therefore, needed to formulate suitable breeding procedures and to foresee the possibility how particular trait could be improved.

In the present study, data on eight quantitative traits were analyzed to assess the extent of induced variability in $\mathrm{M}_{2}$ and $\mathrm{M}_{3}$ generations of the two varieties of chickpea and the control. The extent of variability induced by gamma rays and EMS alone or in combination differed from trait to trait. Study of the direction of shift in mean values of quantitative traits ascertains whether mutation breeding can be restored for the improvement of a trait under study. Although means shifted on either side of the control mean, most of them went towards the positive side for yield and yield contributing traits. Opinions differ regarding the direction of the mutations. Most quantitative traits have a complex genetic determination involving large number of genes interacting with one another, consequently, variation in both the directions is expected. From the results of the present study, it could be concluded that the range of quantitative characters through induced mutations are random, bi-directional and the direction of the mutation depends upon the genotype/character under study and the dose applied. This postulation was also reported by Rao et al. (1988), Waxman and Peck (2003) and Siddiqui et al. (2009). Enlargement in range of variability for yield and its attributes such as pods bearing branches, pods per plant and 100 seed weight for the two varieties of chickpea in $M_{2}$ and $M_{3}$ generations is indicative of the wider scope for selection.

The mean flowering time decreased significantly (approximately by 15 days) with an increase in variability in some mutagenic treatments. The reduction in flowering time accompanied by increase in variability (approximately by 35\%) indicated that variability had been induced in desired direction and would offer the possibility for selecting early flowering mutants in such treatments. Kaul (1980b) suggested that the mutation of two dominant genes to their recessive forms makes for an early flowering in peas. The adverse effect of mutagen treatments on plant height was observed in both varieties. The reduction in plant height was greater in combination treatments as compared to individual mutagenic treatments of gamma rays and EMS in both varieties. The reduction in plant height was reported by Rajput (1974), Yaqoob and Rashid (2001), Khan et al. (2005d) and Wani et al. (2011a) in different crops after mutagenic treatments. However, Singh et al. (2000b) and Arulbalachandran and Mullainathan (2009b) reported an increase in plant height after treatments with gamma rays and EMS in Vigna mungo. Decrease in plant height may result from the inhibition of mitotic divisions (Subba Rao, 1988).

Use of mutations for obtaining early maturing varieties has been a frequent breeding objective (Micke, 1979). The data obtained on days to maturity resulted in a gain in reducing the maturity period by 4 days with $0.3 \%$ EMS and $100 \mathrm{~Gy}$ gamma rays $+0.2 \%$ EMS treatments in $M_{3}$ generation. Being cultivated as a winter season crop in Northern India, chickpea faces chilling and freezing temperatures during vegetative and reproductive growth. Cold stress during reproductive growth of chickpea is 
detrimental to flowering and pod set. Early maturity would be ideal for chickpea to avoid severe cold and attain maximum production. Yaqoob and Rashid (2001) in mungbean, Wang et al. (2003b) in soybean, Shamsuzzaman et al. (2005) in chickpea, Singh et al. (2006) in lentil and Arulbalachandran et al. (2009) in urdbean reported a significant reduction in days to maturity after mutagenic treatments.

The mean number of pods bearing branches and pods per plant increased simultaneously in $M_{2}$ and $M_{3}$ generations, suggesting close correlation between these two traits. The author is of the view that the increase in the number of pods per plant in the present study is obviously due to an increase in the number of flowers. The number of pod sets was higher in the lines, which produced large number of flowers. All these three traits namely, number of pods bearing branches, number of pods per plant and number of flowers seem to be highly correlated. It has been suggested (Rajput, 1974; Sangwan and Singh, 1977; Khan, 1982; Khan and Goyal, 2009; Hiremath et al., 2010; Giri et al., 2010; Wani et. al, 2011b) that an increase in the yield of pulses could be achieved by enhancing pod number. The mean number of seeds per pod seems to be a stable character as most of the treatments with gamma rays and EMS alone, or in combination, did not make much difference in the mean values in both varieties of chickpea. This is probably due to the fact that only $1-2$ seeds can be accommodated in the pod of chickpea. Kumar and Sinha (1989) and Giri et al. (2010) in pigeon pea, Khan et al. (2005d) in chickpea recorded a non-significant difference for the number of seeds per pod after mutagenic treatments.

The character of 100 seed weight is a reliable source of measuring yielding ability in pulses. In the present study, 100 seed weight has shown a very significant increase (approximately by $2 \mathrm{~g} / 100$ seed weight) from the control with most of the treatments of gamma rays and EMS used either singly or in combination in both the varieties of chickpea. This character has been reported to be governed by a relatively smaller number of genes, unlike other polygenic traits (Ghose et al., 1960; Khan, 1990). On the contrary, Jana and Roy (1971), Tickoo and Chandra (1999), Waghmare and Mehra (2000) and Giri et al. (2010) reported the reduction in the mean 100 seed weight.

Seed yield in pulses is a complex trait and is influenced by many other quantitative traits such as pods bearing branches, pods per plant, seeds per pod and 100 seed weight. Seed yield increased by 2-4 g in the variety Pusa-256 (normal yield was $23 \mathrm{~g}$ ) and 3-5 g in the variety BG-1053 (normal yield was $20 \mathrm{~g}$ ) after various mutagen treatments. The mean plant yield in $\mathrm{M}_{2}$ generation, except for higher doses of gamma rays and EMS, employed alone or in combination, and in $M_{3}$ generation showed complete positive trend in the mean values of seed yield per plant with almost all the selected mutagen treatments in both the varieties.

In the present study, increase in mean seed yield in $M_{3}$ over $M_{2}$ generation and the controls could be attributed to effective selection adopted for various yieldcontributing traits in $\mathrm{M}_{2}$ generation. Ramulu (1974) concluded that the mean of the treated population, where no selection had been applied with regard to the specific character under study, tended to go down in comparison to the control. In the present 
investigation, increase in mean seed yield per plant may result from the selection of normal looking plants in $\mathrm{M}_{2}$ which led to elimination of aberrant plants and also due to changes induced at genetic level. Kumar (1972), Jana and Roy (1973) and Tar'an et al. (2004) suggested that the selection process should be delayed until $M_{3}$ or later generations following mutagenic treatment. However, the selection of progenies on the basis of desirable mean and greater variance in $\mathrm{M}_{2}$ was found to be highly useful in the present study. Many other workers have also proposed that effective selection for polygenic traits can be done in early generations even in $\mathrm{M}_{2}$ itself (Sneep, 1977; Kaul and Matta, 1985; Sarker and Sharma, 1988; Tickoo and Chandra, 1999; Singh et al., 2001; Solanki and Sharma, 2002; Sheeba et al., 2003; Arulbalachandran and Mullainathan, 2009b; Giri et al., 2010).

The magnitude of the phenotypic variation, however, does not reveal the relative amounts of heritable (genetic), and non-heritable (non-genetic) components of variation. This was ascertained with the help of some genetic parameters like genotypic coefficient of variation (GCV), heritability in broad sense $\left(\mathrm{h}^{2}\right)$ and genetic advance (GA) as percent of mean. Since the genotypic coefficients of variation and heritability of various quantitative traits indicate the degree of stability to the environmental fluctuations and the potential transmissibility of a trait from parent to offspring and from generation to generation hence their estimates are essential (Kaul and Garg, 1979; Sakin and Yildirim, 2004; Kozgar and Khan, 2009). It is clearly evident from the data that considerable amount of genotypic coefficient of variation was induced by different treatments of gamma rays and EMS alone or in combination. The genotypic coefficient of variation was recorded to be high for yield and yield components, except for seeds per pod, while it was comparatively low for other traits like days to flowering, plant height and days to maturity. Such differential behaviour of different traits was reported earlier by Patel and Shah (1982), Kumar and Dubey (2001), Arulbalachandran and Mullainathan (2009b) and Arulbalachandran et al. (2010). The genotypic coefficient of variation for days to flowering, plant height and days to maturity was higher in $M_{2}$ than $M_{3}$ generation. Shakoor and Haq (1980) reported negligible increase in variability, due to generation advance for some quantitative traits, as a result of stabilization of trait in early generation.

The genetic coefficient of variation measures the range of genetic variability shown by the plant character and helps to compare the genetic variability present in various characters. However, with the help of genetic coefficient of variation alone it is not possible to determine the amount of variation that is heritable. Heritability is of interest to plant breeder as an index of transmissibility and a measure of the value of selection for particular trait. The value of heritability depends on the magnitude of all the components of variance and change in any one of the variance values may affect it. The traits such as the number of pods bearing branches, number of pods, 100-seed weight and total plant yield were found to have a high heritability ( $21 \%$ as compared to normal value $2 \%$ ). However, these estimates were from low to moderate for days to flowering, plant height, days 
to maturity and seeds per pod. In the present study, heritability estimate for yield was high. However, low heritability for yield was reported by various workers in rice (Kaul and Kumar, 1983) and in mungbean (Khan et al., 2004b). The disparity in results could be because heritability is an attribute not only of a trait but also of the population, environment and the circumstances to which the genotypes are subjected. The heritability estimates for number of pods bearing branches, number of pods, 100 seed weight and seed yield per plant in $M_{3}$ generation were higher than in $M_{2}$ generation of the treated population of the two varieties of chickpea. The heritability estimate for seeds per pod was low than the control in both the varieties. The decrease in heritability for seeds per pod indicates that, although genetic variance has increased with the mutagenic treatment, the ratio of its increase was not at par with the total phenotypic variance, which also increased. A rational approach towards the improvement of any crop plant involves selection. Genetic advance is an indicator of the expected genetic progress for a particular trait under suitable selection procedure and consequently carries much significance in selfpollinated crops like chickpea. Estimates of heritability along with genetic advance are usually more helpful than the heritability alone in predicting the resultant effects of selection. This is because the heritability estimates are subjected to interactions between the genotype and environment (Lin et al., 1979; Sakin, 2002; Toker, 2004). The estimated values of genetic advance differed in different mutagenic treatments and also from one trait to another. The traits like pods bearing branches, pods per plant, 100 seed weight and total plant yield have shown a high heritability and genetic advance. The traits possessing a high heritability along with a high genetic advance are more responsive to the effective selection and improvement. Induced mutations in polygenes governing characters can best be inferred by the estimation of heritability and genetic advance, in addition to mean and coefficient of variation, in successive generations of mutagen treated population (Siddiqui et al. 2009).

Results indicate that different traits of the same variety exhibit different response to various mutagens and also their heritable proportion of variance and genetic advance show differential patterns. It is possible that each trait of plant is mutagen specific responsive. This was found in the traits like days to flowering, plant height, days to maturity and seeds per pod where the variability was lower when compared to the traits like yield and yield components. Furthermore, the variability for days to flowering, plant height and days to maturity was lower in $M_{3}$ when compared to $\mathrm{M}_{2}$ generation. However, there was an increase in the variability for yield and yield components in $M_{3}$ comparing to $M_{2}$ generation. These findings implicate that some traits may have a tendency to stabilize sooner than others, depending on selection of mutatants and strategies adopted. Therefore, it is suggested that in the two varieties of chickpea, selection for days to flowering, plant height and days to maturity could be confined to $M_{2}$ generation. The yield and yield components, which have shown increased variability in $\mathrm{M}_{3}$, provide further scope to select more promising lines in later generations. 